Journal of Algebra and Its Applications

(C) World Scientific Publishing Company

\title{
COMPUTING COSET LEADERS AND LEADER CODEWORDS OF BINARY CODES
}

\author{
M. BORGES-QUINTANA* and M.A. BORGES-TRENARD \\ Department of Mathematics, Faculty of Mathematics and Computer Science \\ Universidad de Oriente, Santiago de Cuba, Cuba \\ \{mijail,mborges\}@csd.uo.edu.cu \\ I. MÁRQUEZ-CORBELLA \\ GRACE Project, INRIA Saclay \& LIX, CNRS UMR 7161 - École Polytechnique, 91120 \\ Palaiseau Cedex, France. \\ irene.marquez-corbella@inria.fr \\ E. MARTÍNEZ-MORO \\ Institute of Mathematics IMUVa, University of Valladolid \\ Valladolid, Castilla, Spain \\ edgar@maf.uva.es \\ Received (Day Month Year) \\ Revised (Day Month Year) \\ Communicated by [editor]
}

\begin{abstract}
In this paper we use the Gröbner representation of a binary linear code $\mathcal{C}$ to give efficient algorithms for computing the whole set of coset leaders, denoted by $\mathrm{CL}(\mathcal{C})$ and the set of leader codewords, denoted by $\mathrm{L}(\mathcal{C})$. The first algorithm could be adapted to provide not only the Newton and the covering radius of $\mathcal{C}$ but also to determine the coset leader weight distribution. Moreover, providing the set of leader codewords we have a test-set for decoding by a gradient-like decoding algorithm. Another contribution of this article is the relation stablished between zero neighbours and leader codewords.
\end{abstract}

Keywords: Binary codes; Coset leaders; Test set; Gröbner representation.

AMS Mathematics Subject Classification: 94B05, 13P10

\section{Introduction}

The first goal of this article is to discuss a general algorithm that produces an ordered list of the whole set of coset leaders, denoted by $\mathrm{CL}(\mathcal{C})$, of a given binary code $\mathcal{C}$. This algorithm explains the procedure in $[9, \S 11.7]$ in a more transparent way

*Partially funded by RISC-Linz DK-Doctoral Program.

$\dagger$ Third and fourth authors are partially supported by Spanish MCINN under project MTM201236917-C03-02. 
and it can be adapted to determine coset leader weight distribution, the Newton radius and the covering radius which is one of the most important and studied parameters of a linear code. In [14] some general bounds on the Newton radius for binary linear codes are given. Finding the distribution $\left(\alpha_{0}, \ldots, \alpha_{n}\right)$ of cosets leaders (WDCL) for a code $\mathcal{C}$ is a classic problem in Coding Theory, see for instance [11, Chapter 1, Section 5]. This problem is still unsolved for many family of linear codes even for first-order Reed-Muller codes (see [10]).

Our principal contributions are the efficient computation of the set of all coset leaders and the definition and computation of the set of leader codewords (which is a subset of the set of zero-neighbours), by taking advantage of the additive structure of the cosets. In addition, we proved some properties of this set of leader codewords. Note that the structure described in this paper is related to the monotone structure of the sets of correctable and uncorrectable errors also introduced in $[\underline{8}$, where they describe the minimal uncorrectable errors under the ordering and the so-called larger halves of minimal codewords. Moreover, they use this description to give a gradient-like decoding algorithm. The same approach is considered in [16]. Note that, if the decoding is done using minimum distance decoding, a decoding failure occurs if and only if the true error is not a coset leader. Also solving the $t$-bounded distance decoding problem for a general linear code is related with the knowledge of the coset leaders of the code. Finally the set of coset leaders in linear codes has been also related to the set of minimal support codewords which have been used in maximum likelihood decoding analysis [1]12] and in secret sharing schemes since they describe the minimal access structure [13.

All these problems related to the one that concerns this paper are all considered to be hard computational problems (see for instance [12]) even if preprocessing is allowed [6].

Outline of the paper: In Section 2 we have compiled some basic facts on coding theory and the Gröbner representation of binary linear codes. As for prerequisites, the reader is expected to be familiar with these topics. However we will touch only a few aspect of the theory of Gröbner bases since the paper is written in a "Gröbner bases"-free context. For a deeper discussion of Gröbner representation for linear codes we refer the reader to [5] where recent results and some applications are indicated, in order to get a general picture on the subject we recommend 4 .

In Section 3 we provide an algorithm to compute the set of all coset leaders $(\mathrm{CL}(\mathcal{C}))$. A similar algorithm for computing the set of all coset leaders for a binary code follows intuitively from [9, Chapter 11]. However, the algorithm proposed in this section do not only provide the set $\mathrm{CL}(\mathcal{C})$, but also a Gröbner representation of $\mathcal{C}$ which allows the description of a complete decoding algorithm for $\mathcal{C}$. Moreover, this algorithm is crucial in order to derive an algorithm for the computation of the set of leader codewords. The example presented at the end of this section of a binary linear code with 64 cosets and 118 coset leaders suggests extra applications of the algorithm such as how to obtain the weight distribution of the coset leaders 
or the Newton and Covering radius of a code. These applications do not pose a large additional cost to the proposed algorithm.

Section 4 is devoted to show how the previous algorithm can be adapted to compute a test set for the code which we refer to as leader codewords. Not only do we prove that they are zero neighbours but also that the knowledge of the set of leader codewords can be used to compute all coset leaders corresponding to a given received word.

In the final section we point out where to find out some implementations of the algorithms presented in this paper.

\section{Preliminaries}

By $\mathbb{Z}, \mathbb{K}, \mathbb{K}[\mathbf{X}]$ and $\mathbb{F}_{q}$ we denote the ring of integers, an arbitrary finite field, the polynomial ring in $n$ variables over the field $\mathbb{K}$ and the finite field with $q$ elements.

A linear code $\mathcal{C}$ over $\mathbb{F}_{2}$ of length $n$ and dimension $k$, or an $[n, k]$ binary code for short, is a $k$-dimensional subspace of $\mathbb{F}_{2}^{n}$. We will call the vectors $\mathbf{v}$ in $\mathbb{F}_{2}^{n}$ words and the particular case where $\mathbf{v} \in \mathcal{C}$, codewords. For every vector $\mathbf{y} \in \mathbb{F}_{2}^{n}$ its support is define as its support as a vector in $\mathbb{F}_{2}^{n}$, i.e. $\operatorname{supp}(\mathbf{y})=\left\{i \mid y_{i} \neq 0\right\}$ and its Hamming weight, denoted by $\mathrm{w}_{H}(\mathbf{y})$ as the cardinality of $\operatorname{supp}(\mathbf{y})$.

The Hamming distance, $d_{H}(\mathbf{x}, \mathbf{y})$, between two vectors $\mathbf{x}, \mathbf{y} \in \mathbb{F}_{2}^{n}$ is the number of places where they differ, or equivalently, $d_{H}(\mathbf{x}, \mathbf{y})=\mathrm{w}_{H}(\mathbf{x}-\mathbf{y})$. The minimum distance $d(\mathcal{C})$ of a linear code $\mathcal{C}$ is defined as the minimum weight among all nonzero codewords.

Choose a parity check matrix $H$ for $\mathcal{C}$. The Syndrome of a word $\mathbf{y} \in \mathbb{F}_{2}^{n}$ with respect to the parity check matrix $H$ is the vector $S(\mathbf{y})=H \mathbf{y}^{T} \in \mathbb{F}_{2}^{n-k}$. As the syndrome of a codeword is $\mathbf{0}$, then we have a way to test whether the vector belongs to the code. Moreover, there is a one-to-one correspondence between cosets of $\mathcal{C}$ and values of syndromes.

Definition 2.1. The words of minimal Hamming weight in the cosets of $\mathbb{F}_{2}^{n} / \mathcal{C}$ are the set of coset leaders for $\mathcal{C}$ in $\mathbb{F}_{2}^{n}$. We will denote by $\mathrm{CL}(\mathcal{C})$ the set of coset leaders of the code $\mathcal{C}$ and by $\mathrm{CL}(\mathbf{y})$ the subset of coset leaders corresponding to the coset $\mathcal{C}+\mathbf{y}$. We define the weight of a coset as the smallest Hamming weight among all vectors in the coset, or equivalently the weight of one of its leaders.

The zero vector is the unique coset leader of the code $\mathcal{C}$. Moreover, every coset of weight at most $t$ has a unique coset leader, where $t=\left\lfloor\frac{d(\mathcal{C})-1}{2}\right\rfloor$ is the error-correcting capacity of $\mathcal{C}$ and $\lfloor\cdot\rfloor$ denotes the greatest integer function.

For all $r \in \mathbb{Z}_{\geq 0}$ and $\mathbf{v} \in \mathbb{F}_{2}^{n}$ the set $\mathrm{B}(\mathbf{v}, r):=\left\{\mathbf{w} \in \mathbb{F}_{2}^{n} \mid d_{H}(\mathbf{v}, \mathbf{w}) \leq r\right\}$ is called balls around $\mathbf{v}$ with radius $r$ respect to the Hamming metric. Note that its cardinality is $|\mathrm{B}(\mathbf{v}, r)|=\sum_{i=0}^{r}\left(\begin{array}{c}n \\ i\end{array}\right)$. It is well known that complete minimum distance decoding (CDP) over the code $\mathcal{C}$ has a unique solution for those vectors in the union of the Hamming balls of radius $t$ around the codewords of $\mathcal{C}$.

From now on $\left\{\mathbf{e}_{i} \mid i=1, \ldots, n\right\}$ represents the canonical basis of $\mathbb{F}_{2}^{n}$. The fol- 
lowing theorem gives us a nice relationship between the coset leaders.

Theorem 2.1. Let $\mathbf{w} \in \mathrm{CL}(\mathcal{C})$ such that $\mathbf{w}=\mathbf{y}+\mathbf{e}_{i}$ for some word $\mathbf{y} \in \mathbb{F}_{2}^{n}$ and $i \in \operatorname{supp}(\mathbf{w})$, then $\mathbf{y} \in \mathrm{CL}(\mathcal{C})$.

Proof. See [9, Corollary 11.7.7].

Definition 2.2. The Voronoi region of a codeword $\mathbf{c} \in \mathcal{C}$, denoted by $\mathrm{D}(\mathbf{c})$, is defined as:

$$
\mathrm{D}(\mathbf{c})=\left\{\mathbf{y} \in \mathbb{F}_{2}^{n} \mid d_{H}(\mathbf{y}, \mathbf{c}) \leq d_{H}\left(\mathbf{y}, \mathbf{c}^{\prime}\right) \text { for all } \mathbf{c}^{\prime} \in \mathcal{C} \backslash\{\mathbf{0}\}\right\} .
$$

Note that the set of Voronoi regions of a binary code $\mathcal{C}$ covers the space $\mathbb{F}_{2}^{n}$. However, some points of $\mathbb{F}_{2}^{n}$ may be contained in several regions. Furthermore, the Voronoi region of the all-zero codeword $\mathrm{D}(\mathbf{0})$ coincides with the set of coset leaders of $\mathcal{C}$, i.e. $\mathrm{D}(\mathbf{0})=\mathrm{CL}(\mathcal{C})$.

Definition 2.3. A test-set $\mathcal{T}$ for a given binary code $\mathcal{C}$ is a set of codewords such that every word $\mathbf{y}$ either lies in the Voronoi region of the all-zero vector, $\mathrm{D}(\mathbf{0})$, or there exists $\mathbf{t} \in \mathcal{T}$ such that $\mathrm{w}_{H}(\mathbf{y}-\mathbf{t})<\mathrm{w}_{H}(\mathbf{y})$.

We define the following characteristic crossing function: $\boldsymbol{\Delta}: \mathbb{F}_{2}^{s} \longrightarrow \mathbb{Z}^{s}$ which replace the class of 0,1 by the same symbols regarded as integers. This map will be used with matrices and vectors acting coordinate-wise.

Let $\mathbf{X}$ denotes $n$ variables $x_{1}, \ldots, x_{n}$ and let $\mathbf{a}=\left(a_{1}, \ldots, a_{n}\right)$ be an $n$-tuple of elements of the field $\mathbb{F}_{2}$. We will adopt the following notation:

$$
\mathbf{X}^{\mathbf{a}}:=x_{1}^{\mathbf{\Delta} a_{1}} \cdots x_{n}^{\mathbf{\Delta} a_{n}} \in \mathbb{K}[\mathbf{X}]
$$

This relationship enable us to go back to the usual definition of terms in $\mathbb{K}[\mathbf{X}]$.

Definition 2.4. A Gröbner representation of an $[n, k]$ binary linear code $\mathcal{C}$ is a pair $(\mathcal{N}, \phi)$ where:

- $\mathcal{N}$ is a transversal of the cosets in $\mathbb{F}_{2}^{n} / \mathcal{C}$ (i.e. one element of each coset) verifying that $\mathbf{0} \in \mathcal{N}$ and for each $\mathbf{n} \in \mathcal{N} \backslash\{\mathbf{0}\}$ there exists an $\mathbf{e}_{i}$ with $i \in\{1, \ldots, n\}$ such that $\mathbf{n}=\mathbf{n}^{\prime}+\mathbf{e}_{i}$ with $\mathbf{n}^{\prime} \in \mathcal{N}$.

- $\phi: \mathcal{N} \times\left\{\mathbf{e}_{i}\right\}_{i=1}^{n} \longrightarrow \mathcal{N}$ is a function called Matphi function that maps each pair $\left(\mathbf{n}, \mathbf{e}_{i}\right)$ to the element of $\mathcal{N}$ representing the coset that contains $\mathbf{n}+\mathbf{e}_{i}$.

The ideal $I(\mathcal{C})$ associated with a binary code $\mathcal{C}$ is

$$
I(\mathcal{C})=\left\langle\mathbf{X}^{\mathbf{w}_{1}}-\mathbf{X}^{\mathbf{w}_{2}} \mid \mathbf{w}_{1}-\mathbf{w}_{2} \in \mathcal{C}\right\rangle \subseteq \mathbb{K}[\mathbf{X}]
$$

Note that $I(\mathcal{C})$ is a zero-dimensional ideal since the quotient $\operatorname{ring} R=\mathbb{K}[\mathbf{X}] / I(\mathcal{C})$ is a finite dimensional vector space (i.e. $\operatorname{dim}_{\mathbb{K}}(R)<\infty$ ). Moreover, its dimension is equal to the number of cosets in $\mathbb{F}_{2}^{n} / \mathcal{C}$. 
Therefore, the word Gröbner is not casual. Indeed, if we consider the binomial ideal $I(\mathcal{C})$ and a total degree ordering $\prec$, and we compute the reduced Gröbner basis $\mathcal{G}$ of $I(\mathcal{C})$ w.r.t. $\prec$. Then we can take $\mathcal{N}$ as the vectors $\mathbf{w}$ such that $\mathbf{X}^{\mathbf{w}}$ is a standard monomial module $\mathcal{G}$. Moreover, the function Matphi can be seen as the multiplication tables of the standard monomials times the variables $x_{i}$ modulo the ideal $I_{2}(\mathcal{C})$. Note that the Matphi structure is independent of the particular chosen set $\mathcal{N}$ of representative elements of the quotient ring $\mathbb{F}_{2}^{n} / \mathcal{C}$. See 415 for a more general treatment of these concepts.

\section{Computing the set of coset leaders}

Definition 3.1. An ordering $\prec$ on $\mathbb{F}_{2}^{n}$ is a weight compatible ordering if for any vectors $\mathbf{a}, \mathbf{b} \in \mathbb{F}_{2}^{n}$ we say $\mathbf{a} \prec \mathbf{b}$ if

$$
\mathrm{w}_{H}(\mathbf{a})<\mathrm{w}_{H}(\mathbf{b}) \text {, or if, } \mathrm{w}_{H}(\mathbf{a})=\mathrm{w}_{H}(\mathbf{b}) \text { and } \boldsymbol{\Delta} \mathbf{a} \prec_{1} \boldsymbol{\Delta} \mathbf{b}
$$

where $\prec_{1}$ is any admissible order on $\mathbb{N}^{n}$, i.e. we will require that $\prec_{1}$ have the following additional properties:

(1) For any vector $\mathbf{u} \in \mathbb{N}^{n} \backslash\{\mathbf{0}\}, \mathbf{0} \prec_{1} \mathbf{u}$ and,

(2) For any vectors $\mathbf{u}, \mathbf{v}, \mathbf{w} \in \mathbb{N}^{n}$, if $\mathbf{u} \prec_{1} \mathbf{v}$, then $\mathbf{u}+\mathbf{w} \prec_{1} \mathbf{v}+\mathbf{w}$.

Note that a weight compatible ordering $\prec$ is in general not an admissible ordering on $\mathbb{F}_{2}^{n}$. However, a weight compatible ordering $\prec$ on $\mathbb{F}_{2}^{n}$ satisfies:

- $\prec$ is a noetherian-ordering since every strictly decreasing sequence in $\mathbb{F}_{2}^{n}$ eventually terminates (due to the finiteness of the set $\mathbb{F}_{2}^{n}$ ).

- for every pair $\mathbf{a}, \mathbf{b} \in \mathbb{F}_{2}^{n}$, if $\operatorname{supp}(\mathbf{a}) \subset \operatorname{supp}(\mathbf{b})$, then $\mathbf{a} \prec \mathbf{b}$.

Moreover, for every vector $\mathbf{a} \in \mathbb{F}_{2}^{n}$ we have that $\operatorname{deg}\left(\mathbf{X}^{\mathbf{a}}\right)=\mathrm{w}_{H}(\mathbf{a})$, that is, a weight compatible ordering on $\mathbb{F}_{2}^{n}$ can be viewed as a total degree ordering on $\mathbb{K}[\mathbf{X}]$.

Definition 3.2. We define the object List is an ordered set of elements in $\mathbb{F}_{2}^{n}$ w.r.t. a weight compatible order $\prec$ verifying the following properties:

(1) $\mathbf{0} \in$ List.

(2) If $\mathbf{v} \in$ List and $\mathrm{w}_{H}(\mathbf{v})=\mathrm{w}_{H}(N(\mathbf{v}))$ then $\left\{\mathbf{v}+\mathbf{e}_{i} \mid i \notin \operatorname{supp}(\mathbf{v})\right\} \subset$ List, where $N(\mathbf{v})=\min _{\prec}\{\mathbf{w} \mid \mathbf{w} \in$ List $\cap(\mathcal{C}+\mathbf{v})\}$.

We denote by $\mathcal{N}$ the set of distinct $N(\mathbf{v})$ with $\mathbf{v} \in$ List.

Remark 3.1. Observe that if the second condition of Definition 3.2 holds for $\mathbf{v} \in \mathbb{F}_{2}^{n}$ then $\mathbf{v} \in \mathrm{CL}(\mathcal{C})$. In particular, when $\mathbf{v}$ is the first element of List that belongs to $\mathcal{C}+\mathbf{v}$, then $N(\mathbf{v})=\mathbf{v}$.

Next theorem states that the object List includes the set of coset leaders of a given binary linear code.

Theorem 3.1. Let $\mathbf{w} \in \mathbb{F}_{2}^{n}$. If $\mathbf{w} \in \mathrm{CL}(\mathcal{C})$ then $\mathbf{w} \in$ List. 
Proof. We will proceed by induction on $\mathbb{F}_{2}^{n}$ with a weight compatible ordering $\prec$.

By definition, the statement is true for $\mathbf{0} \in \mathbb{F}_{2}^{n}$. Now for the inductive step, we assume that the desired property is true for any word $\mathbf{u} \in \mathrm{CL}(\mathcal{C})$ smaller than an arbitrary but fixed $\mathbf{w} \in \mathrm{CL}(\mathcal{C}) \backslash\{\mathbf{0}\}$ w.r.t. $\prec$, i.e.

$$
\text { if } \mathbf{u} \in \mathrm{CL}(\mathcal{C}) \text { and } \mathbf{u} \prec \mathbf{w} \text { then } \mathbf{u} \in \text { List, }
$$

and show that this implies that $\mathbf{w} \in$ List.

First note that $\mathbf{w}$ can be written as $\mathbf{w}=\mathbf{v}+\mathbf{e}_{i}$ with $i \in \operatorname{supp}(\mathbf{w})$ and $i \notin \operatorname{supp}(\mathbf{v})$, or equivalently $\operatorname{supp}(\mathbf{v}) \subset \operatorname{supp}(\mathbf{w})$, i.e. $\mathbf{v} \prec \mathbf{w}$. Moreover, since $\mathbf{w} \in \operatorname{CL}(\mathcal{C})$, then by Theorem $2.1 \mathbf{v}$ also belongs to $\mathrm{CL}(\mathcal{C})$, thus $\mathrm{w}_{H}(\mathbf{v})=\mathrm{w}_{H}(N(\mathbf{v}))$. So, if we invoke the induction hypothesis we have that $\mathbf{v} \in$ List. We now apply property 2 of Definition 3.2 which gives as claimed, that $\mathbf{w}=\mathbf{v}+\mathbf{e}_{i} \in$ List.

Theorem 3.1 and its proof suggest Algorithm 1 for computing the whole set of coset leaders of a given binary code $\mathcal{C}$.

The subfunctions used in Algorithm 1 are:

- InsertNext[t, Listing], adds to Listing all the sums $\mathbf{t}+\mathbf{e}_{k}$ with $k \notin \operatorname{supp}(\mathbf{t})$, removes duplicates and keeps Listing in increasing order w.r.t. the ordering $\prec$.

- NextTerm[Listing], returns the first element from Listing and deletes it. If Listing is empty returns $\emptyset$.

- Member $[\mathrm{obj}, G]$, returns the position $j$ of obj in $G$ if obj $\in G$ and false otherwise.

Remark 3.2. In Algorithm 1, first we perform subroutine $\mathbf{t}=$ NextTerm[Listing] where the element $\mathbf{t}$ is deleted from the set Listing. Then subroutine InsertNext[t, Listing] is carried out which inserts in Listing all the elements of the form:

$$
\mathbf{t}^{\prime}=\mathbf{t}+\mathbf{e}_{k} \text { with } k \notin \operatorname{supp}(\mathbf{t}) \text {, i.e. } \mathbf{t}^{\prime} \succ \mathbf{t} .
$$

Therefore all the new elements inserted in Listing are greater than those that have already been deleted from it with respect to $\prec$.

Theorem 3.2. Algorithm 1 computes the set of coset leaders of a given binary code $\mathcal{C}$ and its corresponding Matphi function.

Proof. We build the set List formed by all the words inserted in the object Listing during Algorithm 1 Let us first prove that this new set is well defined according to Definition 3.2. By Step 1, $0 \in$ List verifying property 1 of Definition 3.2. In Step 4 the syndrome of $\mathbf{t}=$ NextTerm[Listing] is computed, then we have two possible cases based on the outcome of Step 5:

(1) If $j=$ false then the $\operatorname{coset} \mathcal{C}+\mathbf{t}$ has not yet been considered. Thus, according to Remark 3.1, we have that $N(\mathbf{t})=\mathbf{t}$ and Step $\mathbf{1 7}$ guarantees property 2 of Definition 3.2 


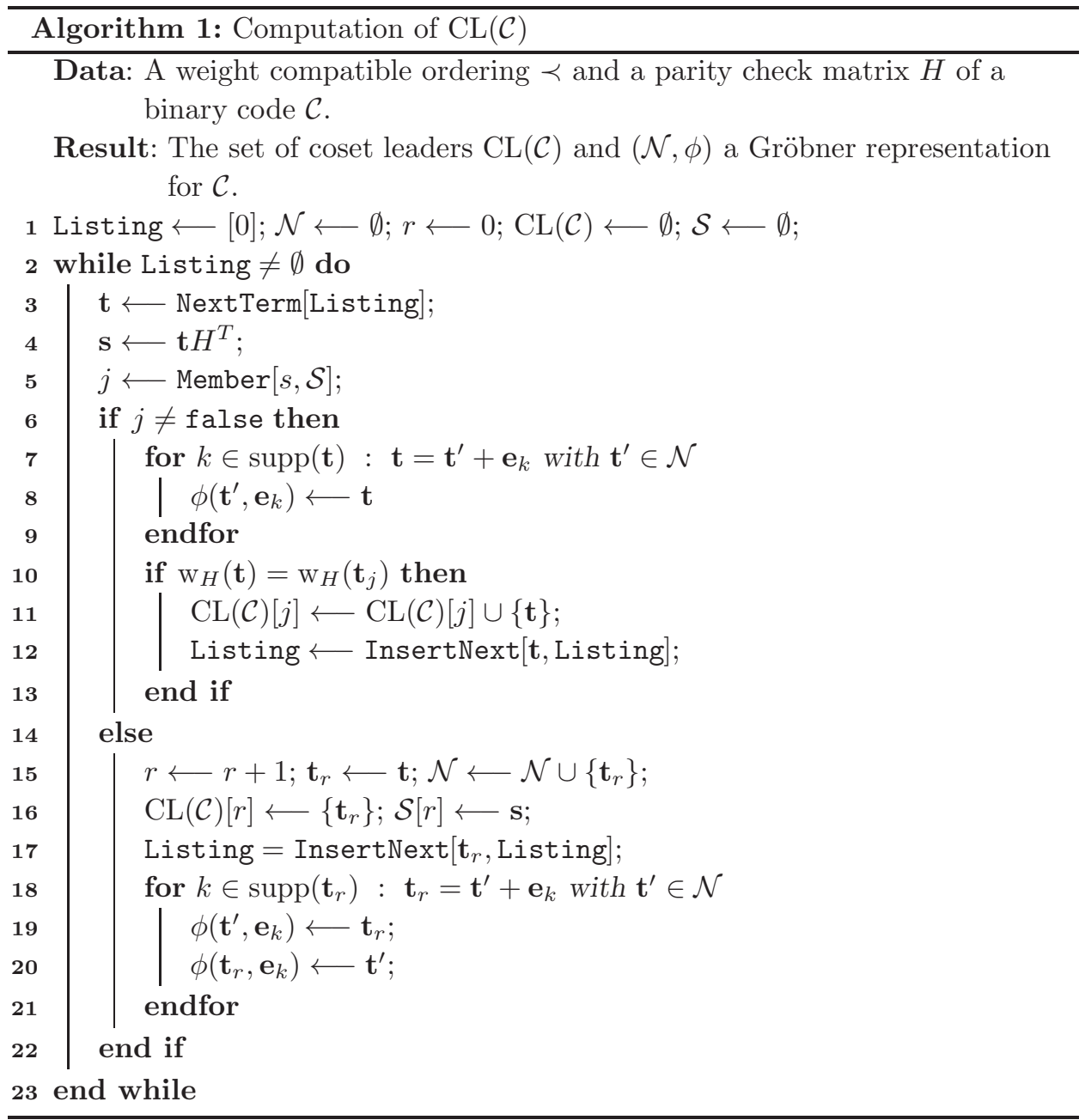

(2) On the other hand, if $j \neq \mathrm{false}$, then the element $N(\mathbf{t})=\mathbf{t}_{j}$ has already been computed. However, if $\mathbf{t} \in \mathrm{CL}(\mathcal{C})$, or equivalently, $\mathrm{w}_{H}(\mathbf{t})=\mathrm{w}_{H}\left(\mathbf{t}_{j}\right)$, then Step 12 certified property 2 .

Therefore Algorithm 1 construct the object List in accordance with Definition 3.2

Furthermore, on one hand Step $\mathbf{1 1}$ and Step $\mathbf{1 6}$ assure the computation of the complete set of coset leaders of the given code; and on the other hand Step 8, Step 19 and Step 20 compute the Matphi function. Note that Step 18 is necessary since the first case above ensures that $N\left(\mathbf{t}_{r}\right)=\mathbf{t}_{r}$ so by Theorem $2.1 \mathbf{t}^{\prime} \in \mathrm{CL}(\mathcal{C})$. But $\mathbf{t}_{r}=\mathbf{t}^{\prime}+\mathbf{e}_{k}$ with $k \in \operatorname{supp}\left(\mathbf{t}_{r}\right)$ so by Remark $3.2 \mathbf{t}^{\prime} \prec \mathbf{t}_{r}$ has already been considered on the algorithm. Thus, we have actually proved that Algorithm 1 guarantees the desired outputs. 
Finally, notice that the cardinality of the set List is bounded by $n$ times the cardinality of $\mathrm{CL}(\mathcal{C})$ and Step $\mathbf{1 2}$ and Step $\mathbf{1 7}$ guarantee that when the complete set of coset leaders is computed no more elements are inserted in Listing while Step 3 continues deleting elements from it. Thus after a finite number of steps the set Listing get empty. Consequently, Step 2 give the end of the algorithm.

Remark 3.3. Note that Algorithm 1 returns $(\mathcal{N}, \phi)$ that fulfill Definition 2.4 for correctness we refer the reader to [5, Theorem 1]. Furthermore, by definition, those representative of the cosets given by $\mathcal{N}$ are the smallest terms in List w.r.t. $\prec$.

Remark 3.4. Algorithm 1 has some similarities with the approach that can be deduced from [9, §11.7] for computing the whole set of coset leaders in a binary code. First of all, Algorithm 1 explains the algorithm in [9, §11.7] in a more transparent way. For more details, let us consider the partial order defined by $\mathbf{x} \leq \mathbf{y}$ if $\operatorname{supp}(\mathbf{x}) \subseteq \operatorname{supp}(\mathbf{y})$ where $\mathbf{x}, \mathbf{y}$ are two elements in $\mathbb{F}_{2}^{n}$. One can use this partial order on $\mathbb{F}_{2}^{n}$ to define a partial order on the set of cosets of a binary code $\mathcal{C}$ as follows: let $\mathbf{y}_{1}+\mathcal{C}$ and $\mathbf{y}_{2}+\mathcal{C}$ be two different cosets of $\mathcal{C}$ then

$\mathbf{y}_{1}+\mathcal{C} \leq \mathbf{y}_{2}+\mathcal{C} \Longleftrightarrow \exists \mathbf{x}_{1} \in \mathrm{CL}\left(\mathbf{y}_{1}\right)$ and $\exists \mathbf{x}_{2} \in \mathrm{CL}\left(\mathbf{y}_{2}\right)$ such that $\mathbf{x}_{1} \leq \mathbf{x}_{2}$.

It is shown in [9] that we can order the different cosets of a binary code $\mathcal{C}$ as a tree from with root $\mathcal{C}$ and in each edge of the tree one unit is added to the weight of the coset leader with respect to the weight of its descendants.

Therefore, both algorithms coincide in the incremental weight order applied to provide the set $\mathrm{CL}(\mathcal{C})$ but our approach has the following advantages:

(1) Algorithm 1 also returns the additive table $\phi$ associated to the addition of a unit vector to any coset. This tool is fundamental for dealing with decoding.

(2) As it is shown in the next Remark, with similar ideas to our approach, the non-binary case could be also solved.

(3) Moreover, as we will see in Section 4 our algorithm allows the computation of a test-set, which is a much more smaller structure than $\mathrm{CL}(\mathcal{C})$ but which could be used to solve the same problems. Also in this paper it is proven that it is a subset of the so called set of zero-neighbors and it contains any minimal test set according to the cardinality.

Remark 3.5. Also the same idea could be implemented in the most general case of linear codes over $\mathbb{F}_{q}^{n}$, with $q=p^{r}$ and $p$ a prime. If we define for $\mathbf{x}$ and $\mathbf{y}$ in $\mathbb{F}_{q}^{n}$, define $\mathbf{x} \leq \mathbf{y}$ provided that $\operatorname{supp}\left(\mathbf{x}_{i}\right) \subseteq \operatorname{supp}\left(\mathbf{y}_{i}\right)$ for all $i=1, \ldots, n$ where $\mathbf{x}_{i}$ is the $p$-adic expansion of the $i$ th component of $\mathbf{x}$. In this case the ideas in [5] could be used to compute a complete set of coset representatives with an analogous incremental structure with respect to the generalized support (but not with respect the the coset weights) and its additive table $\phi$. Take notice that most of the chosen coset representatives may not be coset leaders if the weight of the coset is greater than the error-correcting capability of the code. On those cosets where the chosen 
representative is not a coset leader a descendant property could be also defined to find the coset leaders. However, other results of this paper as leader codewords and coset leaders can not be straightforward deduced from our approach to the $q$-ary case.

Example 3.1. Consider the $[n=10, k=4, d=4]$ binary code $\mathcal{C}$ defined by the following parity check matrix:

$$
H_{\mathcal{C}}=\left(\begin{array}{llllllllll}
1 & 0 & 0 & 0 & 1 & 0 & 0 & 0 & 0 & 0 \\
1 & 0 & 1 & 1 & 0 & 1 & 0 & 0 & 0 & 0 \\
1 & 1 & 0 & 1 & 0 & 0 & 1 & 0 & 0 & 0 \\
1 & 1 & 1 & 0 & 0 & 0 & 0 & 1 & 0 & 0 \\
1 & 1 & 1 & 1 & 0 & 0 & 0 & 0 & 1 & 0 \\
1 & 1 & 1 & 1 & 0 & 0 & 0 & 0 & 0 & 1
\end{array}\right) \in \mathbb{F}_{2}^{6 \times 10}
$$

Algorithm 1 returns the whole set of coset leaders of $\mathcal{C}$ described in Table 1 ordered w.r.t. the degree reverse lexicographic order $\prec$. We denote by $\mathrm{CL}(\mathcal{C})_{i}^{j}$ the $j$-th element of the set of coset leaders of weight $i$.

The main difference between this paper and previous works is the consideration of all coset leaders and not just those belonging to $\mathcal{N}$. Note that no subword of two elements of $\mathbf{y}=\mathbf{e}_{4}+\mathbf{e}_{5}+\mathbf{e}_{6} \in \mathrm{CL}(\mathcal{C})_{3}^{2}$ is part of $\mathcal{N}$, i.e. $\mathbf{e}_{4}+\mathbf{e}_{5} \in \mathrm{CL}(\mathcal{C})_{2}^{7}$, $\mathbf{e}_{4}+\mathbf{e}_{6} \in \mathrm{CL}(\mathcal{C})_{2}^{13}$ and $\mathbf{e}_{5}+\mathbf{e}_{6} \in \mathrm{CL}(\mathcal{C})_{2}^{1}$ do not lie in $\mathcal{N}$. Therefore the importance of the second property of the Definition 3.2 to obtain the complete set of coset leaders.

Algorithm 1 could be adapted without incrementing the complexity to get more information such as:

- The Newton radius $\nu(\mathcal{C})$ of a binary code $\mathcal{C}$ is the largest weight of any error vector that can be uniquely corrected, or equivalently, $\nu(\mathcal{C})$ is the largest value among the cosets with only one coset leader. In our example it suffice to analyze the last element of the list $\mathrm{CL}(\mathcal{C})$ to obtain the coset of highest weight which contains only one leader, i.e. $\nu(\mathcal{C})=3$ since $\mathrm{CL}(\mathcal{C})_{3}^{23}=\left[\mathbf{e}_{5}+\mathbf{e}_{9}+\mathbf{e}_{10}\right]$.

- The covering radius $\rho(\mathcal{C})$ of a binary code $\mathcal{C}$ is the smallest integer $s$ such that $\mathbb{F}_{2}^{n}$ is the union of the spheres of radius $s$ centered at the codewords of $\mathcal{C}$, i.e. $\rho(\mathcal{C})=\max _{\mathbf{y} \in \mathbb{F}_{2}^{n}} \min _{\mathbf{c} \in \mathcal{C}} d_{H}(\mathbf{y}, \mathbf{c})$. It is well known that $\rho(\mathcal{C})$ is the weight of the coset of largest weight. Likewise, in our example $\rho(\mathcal{C})=3$ since $\mathrm{CL}(\mathcal{C})_{3}^{23}=\left[\mathbf{e}_{5}+\mathbf{e}_{9}+\mathbf{e}_{10}\right]$ is the coset of highest weight.

- The Weight Distribution of the Coset Leaders of a binary code $\mathcal{C}$ is the list WDCL $=\left(\alpha_{0}, \ldots, \alpha_{n}\right)$ where $\alpha_{i}$ with $1 \leq i \leq n$ is the number of cosets with coset leaders of weight $i$. Note that the set $\mathcal{N}$ is enough to compute this parameter. It is clear that

$$
\mathrm{WDCL}=[1,10,30,23,0,0,0,0,0,0] .
$$




\begin{tabular}{|c|c|}
\hline \multicolumn{2}{|r|}{ Coset Leaders CL $(\mathcal{C})$} \\
\hline $\mathrm{CL}(\mathcal{C})_{0}$ & {$[0]$} \\
\hline $\mathrm{CL}(\mathcal{C})_{1}$ & {$\left[\mathbf{e}_{1}\right],\left[\mathbf{e}_{2}\right],\left[\mathbf{e}_{3}\right],\left[\mathbf{e}_{4}\right],\left[\mathbf{e}_{5}\right],\left[\mathbf{e}_{6}\right],\left[\mathbf{e}_{7}\right],\left[\mathbf{e}_{8}\right],\left[\mathbf{e}_{9}\right],\left[\mathbf{e}_{10}\right]$,} \\
\hline $\mathrm{CL}(\mathcal{C})_{2}$ & $\begin{array}{l}{\left[\mathbf{e}_{1}+\mathbf{e}_{2}, \mathbf{e}_{5}+\mathbf{e}_{6}\right],\left[\mathbf{e}_{1}+\mathbf{e}_{3}, \mathbf{e}_{5}+\mathbf{e}_{7}\right],\left[\mathbf{e}_{1}+\mathbf{e}_{4}, \mathbf{e}_{5}+\mathbf{e}_{8}\right],} \\
{\left[\mathbf{e}_{1}+\mathbf{e}_{5}, \mathbf{e}_{2}+\mathbf{e}_{6}, \mathbf{e}_{3}+\mathbf{e}_{7}, \mathbf{e}_{4}+\mathbf{e}_{8}\right],\left[\mathbf{e}_{1}+\mathbf{e}_{6}, \mathbf{e}_{2}+\mathbf{e}_{5}\right],} \\
{\left[\mathbf{e}_{1}+\mathbf{e}_{7}, \mathbf{e}_{3}+\mathbf{e}_{5}\right],\left[\mathbf{e}_{1}+\mathbf{e}_{8}, \mathbf{e}_{4}+\mathbf{e}_{5}\right],\left[\mathbf{e}_{1}+\mathbf{e}_{9}\right],\left[\mathbf{e}_{1}+\mathbf{e}_{10}\right],} \\
{\left[\mathbf{e}_{2}+\mathbf{e}_{3}, \mathbf{e}_{6}+\mathbf{e}_{7}\right],\left[\mathbf{e}_{2}+\mathbf{e}_{4}, \mathbf{e}_{6}+\mathbf{e}_{8}\right],\left[\mathbf{e}_{2}+\mathbf{e}_{7}, \mathbf{e}_{3}+\mathbf{e}_{6}\right],} \\
{\left[\mathbf{e}_{2}+\mathbf{e}_{8}, \mathbf{e}_{4}+\mathbf{e}_{6}\right],\left[\mathbf{e}_{2}+\mathbf{e}_{9}\right],\left[\mathbf{e}_{2}+\mathbf{e}_{10}\right],} \\
{\left[\mathbf{e}_{3}+\mathbf{e}_{4}, \mathbf{e}_{7}+\mathbf{e}_{8}\right],\left[\mathbf{e}_{3}+\mathbf{e}_{8}, \mathbf{e}_{4}+\mathbf{e}_{7}\right],\left[\mathbf{e}_{3}+\mathbf{e}_{9}\right],\left[\mathbf{e}_{5}+\mathbf{e}_{9}\right],} \\
{\left[\mathbf{e}_{3}+\mathbf{e}_{10}\right],\left[\mathbf{e}_{4}+\mathbf{e}_{9}\right],\left[\mathbf{e}_{4}+\mathbf{e}_{10}\right],\left[\mathbf{e}_{5}+\mathbf{e}_{0}\right],\left[\mathbf{e}_{6}+\mathbf{e}_{10}\right],\left[\mathbf{e}_{7}+\mathbf{e}_{9}\right],} \\
{\left[\mathbf{e}_{5}+\mathbf{e}_{10}\right],\left[\mathbf{e}_{6}+\mathbf{e}_{9}\right],\left[\mathbf{e}_{8}+\mathbf{e}_{10}\right],\left[\mathbf{e}_{9}+\mathbf{e}_{10}\right],}\end{array}$ \\
\hline $\mathrm{CL}(\mathcal{C})_{3}$ & 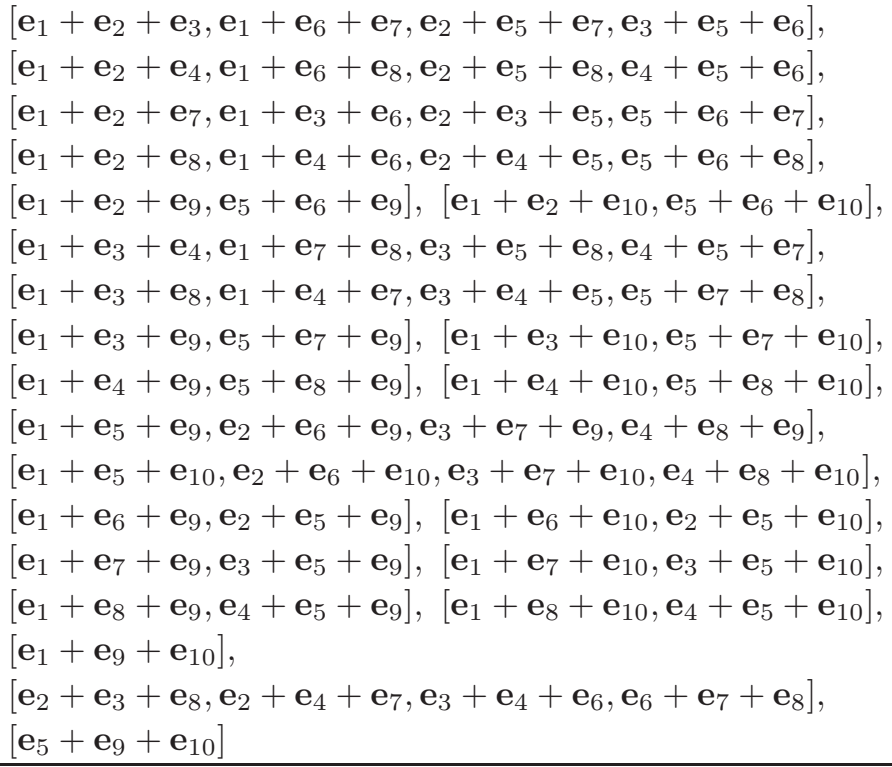 \\
\hline
\end{tabular}

Table 1. Set of coset-leaders of Example 3.1

- The number of coset leaders in each coset :

$$
\sharp(\mathrm{CL})=\left[\begin{array}{l}
1, \\
1,1,1,1,1,1,1,1,1,1, \\
2,2,2,4,2,2,2,1,1,2,2,2,2,1,1,2,2,1,1,1,1,1,1,1,1,1,1,1,1,1, \\
4,4,4,4,2,2,4,4,2,2,2,2,4,4,2,2,2,2,2,2,1,4,1
\end{array}\right]
$$

Note that there are 30 of the 64 cosets where the Complete Decoding Problem (CDP) has a unique solution. It is also interesting to note that among the cosets with one leaders there are more cosets exceeding the error correction capacity (19) than achieving such capacity (11). 


\subsection{Complexity Analysis}

The next theorem states an upper bound for the number of iterations that Algorithm 1 will perform.

Theorem 3.3. Algorithm 1 computes the set of coset leaders of a given binary code $\mathcal{C}$ of length $\mathbf{n}$ after at most $\mathbf{n}|\mathrm{CL}(\mathcal{C})|$ iterations.

Proof. Let List be the set constructed in the proof of Theorem 3.2. Notice that by looking how Algorithm 1 is constructed, the number of iterations is exactly the size of List. Moreover note that we can write List as the following set

$$
\text { List }=\left\{\mathbf{w}+\mathbf{e}_{i} \mid \mathbf{w} \in \mathrm{CL}(\mathcal{C}) \text { and } i \in\{1, \ldots, n\}\right\} .
$$

Therefore it is clear that the size of List is bounded by $\mathbf{n}|\mathrm{CL}(\mathcal{C})|$.

\section{Remark 3.6.}

(1) We can proceed analogously to the previous proof to estimate the required memory space which is $\mathcal{O}(\mathbf{n}|\mathrm{CL}(\mathcal{C})|)$. In the best case, $\mathcal{O}(|\mathrm{CL}(\mathcal{C})|)$ of memory space is needed, thus Algorithm 1 is near the optimal case when considering memory requirements. However the order of the set $\mathrm{CL}(\mathcal{C})$ is exponential on the codimension of the code, i.e. $\mathcal{O}\left(2^{n-k}\right)$, so this method is impractical for large codes.

(2) Algorithm 1 generates at most $\mathbf{n}|\mathrm{CL}(\mathcal{C})|$ words from $\mathbb{F}_{2}^{n}$ to compute the set of all coset leaders. Therefore, the proposed algorithm has near-optimal performance and significantly reduced complexity.

Note that the statement $\mathrm{B}(\mathbf{c}, e) \cap \mathrm{B}(\hat{\mathbf{c}}, e)=\emptyset$ holds true for all $\mathbf{c}, \hat{\mathbf{c}} \in \mathcal{C}$ with $\mathbf{c} \neq \hat{\mathbf{c}}$ if and only if $2 e+1 \leq d(\mathcal{C})$ is valid. Moreover, $\mathbb{F}_{2}^{n}=\cup_{\mathbf{c} \in \mathcal{C}} \mathrm{B}(\mathbf{c}, e)$ holds true if and only if the covering radius satisfies that $\rho(\mathcal{C}) \leq e$. Therefore the minimum distance and the covering radius of any code are related by $d(\mathcal{C}) \leq 2 \rho(\mathcal{C})+1$.

Lemma 3.1. For any $[n, k]$ binary code $\mathcal{C}$ the following inequality holds:

$$
\sum_{i=0}^{t}\left(\begin{array}{l}
n \\
i
\end{array}\right) \leq|\mathrm{CL}(\mathcal{C})| \leq \sum_{j=0}^{\rho(\mathcal{C})}\left(\begin{array}{l}
n \\
j
\end{array}\right),
$$

where $t$ denotes the error-correcting capacity of $\mathcal{C}$ and $\rho(\mathcal{C})$ its covering radius.

Proof. Let us first prove that every vector $\mathbf{e} \in \mathbb{F}_{2}^{n}$ with $\mathrm{w}_{H}(\mathbf{e}) \leq t$ is a coset leader. Assume to the contrary that there exists a vector $\mathbf{e} \in \mathbb{F}_{2}^{n}$ with $\mathrm{w}_{H}(\mathbf{e}) \leq t$ and $\mathbf{e} \notin \mathrm{CL}(\mathcal{C})$. Hence there is another vector $\hat{\mathbf{e}} \in \mathbb{F}_{2}^{n}$ with $S(\mathbf{e})=S(\hat{\mathbf{e}})$ and $\mathrm{w}_{H}(\hat{\mathbf{e}})<\mathrm{w}_{H}(\mathbf{e})$. Or equivalently, there exists a codeword $\mathbf{e}-\hat{\mathbf{e}} \in \mathcal{C}$ with

$$
\mathrm{w}_{H}(\mathbf{e}-\hat{\mathbf{e}}) \leq \mathrm{w}_{H}(\mathbf{e})+\mathrm{w}_{H}(\hat{\mathbf{e}}) \leq 2 t \leq d(\mathcal{C})-1
$$


which is a contradiction to the definition of the minimum distance of $\mathcal{C}$. Hence, we have actually proved that the number of vectors of weight up to $t$ is a lower bound for the cardinality of the set $\mathrm{CL}(\mathcal{C})$, i.e.

$$
\sum_{i=0}^{t}\left(\begin{array}{c}
n \\
i
\end{array}\right) \leq|\mathrm{CL}(\mathcal{C})| .
$$

Furthermore, by the definition of the covering radius of $\mathcal{C}$, we have that for all $\mathbf{y} \in \mathbb{F}_{2}^{n}$ there exists a codeword $\mathbf{c} \in \mathcal{C}$ such that $\mathrm{d}_{H}(\mathbf{c}, \mathbf{y}) \leq \rho(\mathcal{C})$. In other words, there exists a vector $\mathbf{e} \in \mathbb{F}_{2}^{n}$ such that $\mathrm{w}_{H}(\mathbf{e}) \leq \rho(\mathcal{C})$ and $S(\mathbf{e})=S(\mathbf{y})$. Thus, $\mathrm{w}_{H}(\mathrm{CL}(\mathbf{y})) \leq \rho(\mathcal{C})$ and the lemma holds.

If the above lemma holds with equality then $\mathcal{C}$ is called a perfect code. That is to say, let $\mathcal{C}$ be a linear code with more than one codeword, then $\mathcal{C}$ is a perfect code if and only if $\rho(\mathcal{C})=t$.

\section{Computing a test set}

In this section we show how Algorithm 1 can be adapted to compute a test-set for a binary linear code.

Definition 4.1. The set of leader codewords of a given binary code $\mathcal{C}$ is defined as:

$$
\mathrm{L}(\mathcal{C})=\left\{\mathbf{n}_{1}+\mathbf{n}_{2}+\mathbf{e}_{i} \in \mathcal{C} \backslash\{\mathbf{0}\} \mid i \notin \operatorname{supp}\left(\mathbf{n}_{1}\right) \text { and } \mathbf{n}_{1}, \mathbf{n}_{2} \in \mathrm{CL}(\mathcal{C})\right\}
$$

For efficiency reasons we are just interested in a particular case of the above object, when $\operatorname{supp}\left(\mathbf{n}_{1}+\mathbf{e}_{i}\right) \cap \operatorname{supp}\left(\mathbf{n}_{2}\right)=\emptyset$.

Remark 4.1. The difference between Algorithm 1 and Algorithm 2 are Steps 10-12 from Algorithm 2 .

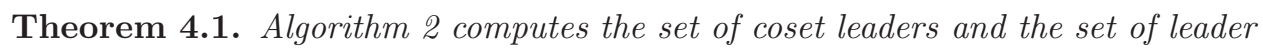
codewords of a given binary code $\mathcal{C}$.

Proof. Taking into account Remark 4.1 and Theorem 3.2 we only need to prove that Algorithm 2 computes the set of leader codewords.

We first observe that all the words inserted in the set $\mathrm{L}(\mathcal{C})$ during Algorithm 2 are leader codewords. These elements are of the type $\mathbf{v}=\mathbf{t}^{\prime}+\mathbf{e}_{i}+\mathbf{t}_{k}$ where $\mathbf{t}=\mathbf{t}^{\prime}+\mathbf{e}_{i}$ and $\mathbf{t}_{k}$ are in the same coset. Moreover we have that

$$
\mathbf{t}^{\prime}, \mathbf{t}_{k} \in \mathrm{CL}(\mathcal{C}), \operatorname{supp}\left(\mathbf{t}^{\prime}\right) \cap \operatorname{supp}\left(\mathbf{t}_{k}\right)=\emptyset \text { and } i \notin \operatorname{supp}\left(\mathbf{t}^{\prime}\right) .
$$

Therefore, by Definition $4.1 \mathrm{v}$ is a leader codeword.

Note that the list Listing is in ascending order w.r.t. $\prec$, therefore in each loop we study all leader codewords of the form $\mathbf{n}_{1}+\mathbf{e}_{i}+\mathbf{n}_{2}$ with $\mathbf{n}_{1}, \mathbf{n}_{2} \leq \mathbf{t}$. The fact that Theorem 3.2 shows that Algorithm 2 computes the whole set $\mathrm{CL}(\mathcal{C})$ proves that all leader codewords are introduced in $\mathrm{L}(\mathcal{C})$. 


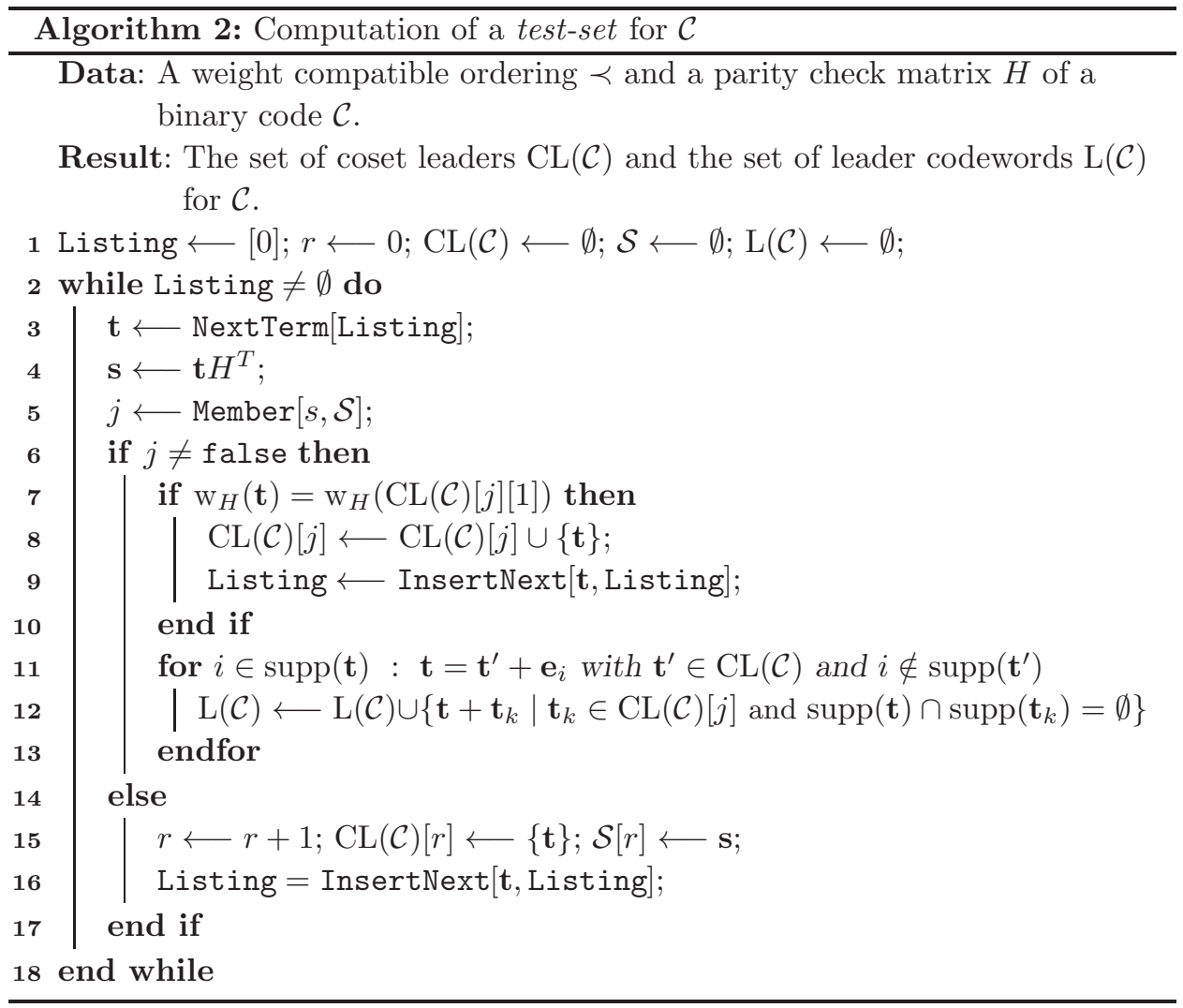

By its construction, Algorithm 2 has the same time complexity as Algorithm 1 The advantage of computing the set of leader codewords is that it helps in solving the same problems as the function Matphi does but with a structure which is considerately smaller.

Definition 4.2. We define the subset $\mathrm{L}^{1}(\mathcal{C})$ of $\mathrm{L}(\mathcal{C})$ as

$$
\mathrm{L}^{1}(\mathcal{C})=\left\{\mathbf{n}_{1}+\mathbf{n}_{2}+\mathbf{e}_{i} \in \mathcal{C} \backslash\{\mathbf{0}\} \mid \begin{array}{c}
i \notin \operatorname{supp}\left(\mathbf{n}_{1}\right), \mathbf{n}_{1} \in \operatorname{CL}(\mathcal{C}), \mathbf{n}_{2} \in \mathcal{N} \\
\text { and } \mathrm{w}_{H}\left(\mathbf{n}_{1}+\mathbf{e}_{i}\right)>\mathrm{w}_{H}\left(\mathbf{n}_{2}\right),
\end{array}\right\} .
$$

Remark 4.2. Note that the condition $\mathrm{w}_{H}\left(\mathbf{n}_{1}+\mathbf{e}_{i}\right)>\mathrm{w}_{H}\left(\mathbf{n}_{2}\right)$ is imposed just to improve the efficiency of computing this set. Therefore $\mathrm{L}(\mathcal{C})$ can be rewritten as

$$
\mathrm{L}(\mathcal{C})=\left\{\begin{array}{l|l}
\mathbf{n}_{1}+\mathbf{n}_{2}+\mathbf{e}_{i} \in \mathcal{C} \backslash\{\mathbf{0}\} & \begin{array}{l}
i \notin \operatorname{supp}\left(\mathbf{n}_{1}\right), \mathbf{n}_{1}, \mathbf{n}_{2} \in \mathrm{CL}(\mathcal{C}) \\
\text { and } \mathrm{w}_{H}\left(\mathbf{n}_{1}+\mathbf{e}_{i}\right)>\mathrm{w}_{H}\left(\mathbf{n}_{2}\right)
\end{array}
\end{array}\right\} .
$$

Thus, the only difference between the sets $\mathrm{L}^{1}(\mathcal{C})$ and $\mathrm{L}(\mathcal{C})$ is that $\mathbf{n}_{2} \in \mathcal{N}$ instead of $\mathbf{n}_{2} \in \mathrm{CL}(\mathcal{C})$. In other words, to the element $\mathbf{n}_{2}$ in $\mathrm{L}^{1}(\mathcal{C})$ is required not only to belong to the set of the coset leaders but also to be the smallest element in its coset according to a fixed weight compatible ordering $\prec$. 
Theorem 4.2. The subset $\mathrm{L}^{1}(\mathcal{C})$ of $\mathrm{L}(\mathcal{C})$ is a test-set for $\mathcal{C}$.

Proof. Let us consider a word $\mathbf{y} \notin \mathrm{CL}(\mathcal{C})$ with $\operatorname{supp}(\mathbf{y})=\left\{i_{1}, \ldots, i_{m}\right\} \subseteq[1, n]$. Thus, there must exist an integer $1 \leq l<m$ such that

$$
\mathbf{n}_{1}:=\mathbf{e}_{i_{1}}+\ldots+\mathbf{e}_{i_{l}} \in \mathrm{CL}(\mathcal{C}) \text { and } \mathbf{n}_{1}+\mathbf{e}_{i_{l+1}} \notin \mathrm{CL}(\mathcal{C}) .
$$

We define $\mathbf{n}_{2}=N\left(\mathbf{n}_{1}+\mathbf{e}_{i_{l+1}}\right)$, i.e. $\mathbf{n}_{2}$ is the smallest element in the coset of $\mathbf{n}_{1}+\mathbf{e}_{i_{l+1}}$ according to a fixed compatible weight ordering $\succ$. Since $\mathbf{n}_{1}+\mathbf{e}_{i_{l+1}} \notin$ $\mathrm{CL}(\mathcal{C})$ we have that $\mathrm{w}_{H}\left(\mathbf{n}_{2}\right)<\mathrm{w}_{H}\left(\mathbf{n}_{1}+\mathbf{e}_{i_{l+1}}\right)$. Thus, $\mathbf{t}=\mathbf{n}_{1}+\mathbf{n}_{2}+\mathbf{e}_{i_{l+1}} \in \mathrm{L}^{1}(\mathcal{C})$.

Without loss of generality we may assume that $\operatorname{supp}\left(\mathbf{n}_{1}+\mathbf{e}_{i_{l+1}}\right) \cap \operatorname{supp}\left(\mathbf{n}_{2}\right)=\emptyset$. Indeed,

- if $i_{l+1} \in \operatorname{supp}\left(\mathbf{n}_{2}\right)$ then, by Theorem 2.1, $\mathbf{n}_{2}+\mathbf{e}_{i_{l+1}} \in \mathrm{CL}(\mathcal{C})$. Moreover

$$
S\left(\mathbf{n}_{2}+\mathbf{e}_{i_{l+1}}\right)=S\left(\mathbf{n}_{1}\right) \text { and } \mathrm{w}_{H}\left(\mathbf{n}_{2}+\mathbf{e}_{i_{l+1}}\right)<\mathrm{w}_{H}\left(\mathbf{n}_{1}\right),
$$

which contradicts the fact that $\mathbf{n}_{1} \in \mathrm{CL}(\mathcal{C})$.

- Otherwise, if there exists $j \in \operatorname{supp}\left(\mathbf{n}_{1}\right) \cap \operatorname{supp}\left(\mathbf{n}_{2}\right)$. Then, we replace the elements $\mathbf{n}_{1}$ and $\mathbf{n}_{2}$ by $\overline{\mathbf{n}_{1}}=\mathbf{n}_{1}+\mathbf{e}_{j}$ and $\overline{\mathbf{n}_{2}}=\mathbf{n}_{2}+\mathbf{e}_{j}$. Note that, by Theorem 2.1. $\overline{\mathbf{n}_{1}}, \overline{\mathbf{n}_{2}} \in \mathrm{CL}(\mathcal{C})$. Furthermore,

$$
\mathrm{w}_{H}\left(\overline{\mathbf{n}_{1}}+\mathbf{e}_{i_{l+1}}\right)>\mathrm{w}_{H}\left(\overline{\mathbf{n}_{2}}\right) \text { and } S\left(\overline{\mathbf{n}_{1}}+\mathbf{e}_{i_{l+1}}\right)=S\left(\overline{\mathbf{n}_{2}}\right) .
$$

Thus we still have that $\mathbf{t}=\overline{\mathbf{n}_{1}}+\overline{\mathbf{n}_{2}}+\mathbf{e}_{i_{l+1}} \in \mathrm{L}^{1}(\mathcal{C})$.

Therefore, $|\operatorname{supp}(\mathbf{t}) \cap \operatorname{supp}(\mathbf{y})| \geq \mathrm{w}_{H}\left(\mathbf{n}_{1}+\mathbf{e}_{i_{l+1}}\right)>\mathrm{w}_{H}\left(\mathbf{n}_{2}\right) \geq|\operatorname{supp}(\mathbf{t}) \cap \operatorname{supp}(\overline{\mathbf{y}})|$ where $\overline{\mathbf{y}}$ denotes the relative complement of $\mathbf{y}$ in $\mathbb{F}_{2}^{n}$, and in consequence, $\mathrm{w}_{H}(\mathbf{y}-$ t) $<\mathrm{w}_{H}(\mathbf{y})$ which completes the proof.

Since $\mathrm{L}^{1}(\mathcal{C}) \subseteq \mathrm{L}(\mathcal{C})$ and by Theorem 4.2 the subset $\mathrm{L}^{1}(\mathcal{C})$ forms a test-set for $\mathcal{C}$, then so does the set $\mathrm{L}(\mathcal{C})$. The following theorem gives a bound for the weight of a leader codeword of a given binary code $\mathcal{C}$.

Theorem 4.3. Let $\mathbf{c} \in \mathrm{L}(\mathcal{C})$ then $\mathrm{w}_{H}(\mathbf{c}) \leq 2 \rho(\mathcal{C})+1$ where $\rho(\mathcal{C})$ is the covering radius of $\mathcal{C}$.

Proof. Let $\mathbf{c} \in \mathrm{L}(\mathcal{C})$ then there exists $\mathbf{n}_{1}, \mathbf{n}_{2} \in \mathrm{CL}(\mathcal{C})$ and $i \notin \operatorname{supp}\left(\mathbf{n}_{1}\right)$ such that $\mathrm{w}_{H}\left(\mathbf{n}_{1}+\mathbf{e}_{i}\right)>\mathrm{w}_{H}\left(\mathbf{n}_{2}\right)$ and $\mathbf{c}=\mathbf{n}_{1}+\mathbf{e}_{i}+\mathbf{n}_{2}$. Applying the definition of covering radius we have that $\mathrm{w}_{H}\left(\mathbf{n}_{1}\right), \mathrm{w}_{H}\left(\mathbf{n}_{2}\right) \leq \rho$, thus $\mathrm{w}_{H}(\mathbf{c}) \leq 2 \rho+1$.

In Algorithm 3 we describe a method to compute the subset $\mathrm{CL}(\mathbf{y})$ of coset leaders corresponding to the $\operatorname{coset} \mathcal{C}+\mathbf{y}$. Note that we first need to achieve the element $N(\mathbf{y})$. We propose to use a Gradient Descent Decoding Algorithm (GDDA) for this purpose. This approach resembles those techniques presented in [3].

Theorem 4.4. Algorithm computes, from the set $\mathrm{L}(\mathcal{C})$, the subset $\mathrm{CL}(\mathbf{y})$ of coset leaders corresponding to the coset $\mathcal{C}+\mathbf{y}$ for a given received vector $\mathbf{y} \in \mathbb{F}_{2}^{n}$. 


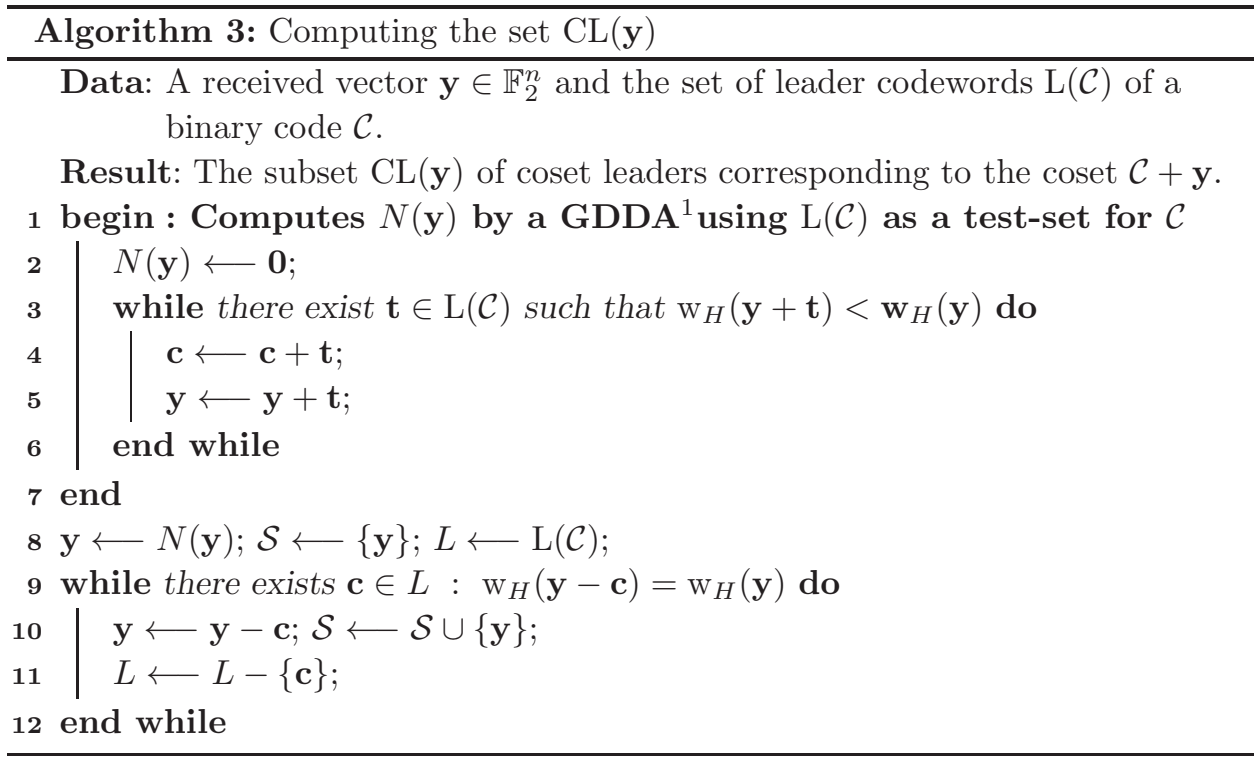

Proof. Let us first prove that every $\mathbf{z} \in \mathrm{CL}(\mathbf{y})$ can be rewritten as $\mathbf{z}=N(\mathbf{y})-\mathbf{c}$ with $\mathbf{c} \in \mathrm{L}(\mathcal{C})$. Let $i \in \operatorname{supp}(\mathbf{z})$ then $\mathbf{z}=\mathbf{n}_{1}+\mathbf{e}_{i}$ with $i \notin \operatorname{supp}\left(\mathbf{n}_{1}\right)$. Hence, by Theorem 2.1, $\mathbf{n}_{1} \in \mathrm{CL}(\mathcal{C})$. Furthermore we have that

$$
S(N(\mathbf{y}))=S(\mathbf{z}) \text { and } \mathrm{w}_{H}\left(\mathbf{n}_{1}\right)<\mathrm{w}_{H}(\mathbf{z})=\mathrm{w}_{H}(N(\mathbf{y})) .
$$

Thus, from the definition of leader codewords, $\mathbf{c}=N(\mathbf{y})+\left(\mathbf{n}_{1}+\mathbf{e}_{i}\right) \in \mathrm{L}(\mathcal{C}) \subseteq \mathcal{C}$, or equivalently, $\mathbf{z}=\mathbf{n}_{1}+\mathbf{e}_{i}=\mathbf{c}-N(\mathbf{y})$ with $\mathbf{y} \in L(\mathcal{C})$.

The proof is completed by noting that Theorem 4.2 guarantees Step 1.

\subsection{Leader codewords and zero neighbours}

In this section we will give a brief review of basic concepts from [1, Section 3] and thus establish the relation between zero neighbours and leader codewords of a binary code $\mathcal{C}$.

Definition 4.3. For any subset $A \subset \mathbb{F}_{2}^{n}$ we define $\mathcal{X}(A)$ as the set of words at Hamming distance 1 from $A$, i.e.

$$
\mathcal{X}(A)=\left\{\mathbf{y} \in \mathbb{F}_{2}^{n} \mid \min \left\{d_{H}(\mathbf{y}, \mathbf{a}): \mathbf{a} \in A\right\}=1\right\} .
$$

We define the boundary of $A$ as $\delta(A)=\mathcal{X}(A) \cup \mathcal{X}\left(\mathbb{F}_{2}^{n} \backslash A\right)$.

Definition 4.4. A nonzero codeword $\mathbf{c} \in \mathcal{C}$ is called zero neighbour if its Voronoi region shares a common boundary with the set of coset leaders, i.e.

$$
\delta(\mathrm{D}(\mathbf{z})) \cap \delta(\mathrm{D}(\mathbf{0})) \neq \emptyset .
$$

\footnotetext{
${ }^{1}$ GDDA is the abbreviation for Gradient Descent Decoding Algorithm
} 
We will denote by $\mathcal{Z}(\mathcal{C})$ the set of all zero neighbours of $\mathcal{C}$ that is to say:

$$
\mathcal{Z}(\mathcal{C})=\{\mathbf{z} \in \mathcal{C} \backslash\{\mathbf{0}\}: \delta(\mathrm{D}(\mathbf{z})) \cap \delta(\mathrm{D}(\mathbf{0})) \neq \emptyset\} .
$$

Note that if $\mathbf{z} \in \mathcal{C} \backslash\{\mathbf{0}\}$ satisfies that $\mathcal{X}(\mathrm{D}(\mathbf{0})) \cap \mathrm{D}(\mathbf{z}) \neq \emptyset$, then $\mathbf{z} \in \mathcal{Z}(\mathcal{C})$. Furthermore $\mathcal{Z}(\mathcal{C})$ is a test-set for $\mathcal{C}$ (see for instance [1, Theorem 3.16]). However the only property of the set $\mathcal{Z}(\mathcal{C})$ that is essential for decoding is

$$
\mathcal{X}(\mathrm{D}(\mathbf{0})) \subseteq \bigcup_{\mathbf{z} \in \mathcal{Z}(\mathcal{C})} \mathrm{D}(\mathbf{z})
$$

Thus, if we restrict the set $\mathcal{Z}(\mathcal{C})$ to a smallest subset verifying the previous property we still have a test-set for $\mathcal{C}$. We will denote such subset of $\mathcal{Z}(\mathcal{C})$ by $\mathcal{Z}_{\text {min }}(\mathcal{C})$. Note that the set $\mathcal{Z}_{\min }(\mathcal{C})$ may be not unique, however its size is well defined.

Theorem 4.5. Let $\mathcal{C}$ be a binary code and $\mathbf{z} \in \mathcal{C} \backslash\{\mathbf{0}\}$. Then the following are equivalent:

(1) $\mathcal{X}(\mathrm{D}(\mathbf{0})) \cap \mathrm{D}(\mathbf{z}) \neq \emptyset$.

(2) $\mathbf{z} \in \mathrm{L}(\mathcal{C})$.

Proof. If $\mathcal{X}(\mathrm{D}(\mathbf{0})) \cap \mathrm{D}(\mathbf{z}) \neq \emptyset$ then there exists $\mathbf{n}_{1} \in \mathrm{D}(\mathbf{0})=\mathrm{CL}(\mathcal{C})$ and $i \notin$ $\operatorname{supp}\left(\mathbf{n}_{1}\right)$ such that $\mathbf{n}_{1}+\mathbf{e}_{i} \in \mathcal{X}(\mathrm{D}(\mathbf{0}))$ and $\mathbf{n}_{1}+\mathbf{e}_{i} \in \mathrm{D}(\mathbf{z})$. In other words,

$$
\mathrm{w}_{H}\left(\mathbf{z}-\left(\mathbf{n}_{1}+\mathbf{e}_{i}\right)\right) \leq \mathrm{w}_{H}\left(\mathbf{c}-\left(\mathbf{n}_{1}+\mathbf{e}_{i}\right)\right) \text { for all } \mathbf{c} \in \mathcal{C} \backslash\{\mathbf{z}\},
$$

or equivalently, $\mathbf{n}_{2}=\mathbf{z}-\left(\mathbf{n}_{1}+\mathbf{e}_{i}\right) \in \mathrm{CL}(\mathcal{C})$ with $\mathbf{z} \in \mathcal{C}$, thus $S\left(\mathbf{n}_{2}\right)=S\left(\mathbf{n}_{1}+\mathbf{e}_{i}\right)$. Furthermore, the special case of $\mathbf{c}=\mathbf{0} \in \mathcal{C} \backslash\{\mathbf{z}\}$ of Equation 4.1 implies that $\mathrm{w}_{H}\left(\mathbf{n}_{2}\right) \leq \mathrm{w}_{H}\left(\mathbf{n}_{1}+\mathbf{e}_{i}\right)$. Therefore, all conditions in Definition 4.1 are verified, i.e. $\mathbf{z}=\mathbf{n}_{1}+\mathbf{n}_{2}+\mathbf{e}_{i} \in \mathrm{L}(\mathcal{C})$.

Conversely, if $\mathbf{z} \in \mathrm{L}(\mathcal{C})$, then $\mathbf{z}$ can be rewritten as $\mathbf{z}=\mathbf{n}_{1}+\mathbf{n}_{2}+\mathbf{e}_{i}$ where

$$
\begin{array}{ll}
\text { (1) } \mathbf{n}_{1}, \mathbf{n}_{2} \in \mathrm{CL}(\mathcal{C}) . & (3) \mathrm{w}_{H}\left(\mathbf{n}_{1}+\mathbf{e}_{i}\right)>\mathrm{w}_{H}\left(\mathbf{n}_{2}\right) . \\
(2) i \notin \operatorname{supp}\left(\mathbf{n}_{1}\right) . & \text { (4) } S\left(\mathbf{n}_{2}\right)=S\left(\mathbf{n}_{1}+\mathbf{e}_{i}\right) .
\end{array}
$$

Now (1) and (2) gives that $\mathbf{n}_{1}+\mathbf{e}_{i} \in \mathcal{X}(\mathrm{D}(\mathbf{0})$ ), whereas (1), (3) and (4) clearly force that $\operatorname{CL}\left(\mathbf{n}_{1}+\mathbf{e}_{i}\right)=\mathbf{n}_{2}$, i.e. $\mathrm{w}_{H}\left(\mathbf{n}_{2}\right) \leq \mathrm{w}_{H}\left(\mathbf{n}_{1}+\mathbf{e}_{i}+\mathbf{c}\right)$ for all $\mathbf{c} \in \mathcal{C}$, or equivalently, $\mathbf{n}_{1}+\mathbf{e}_{i} \in \mathrm{D}(\mathbf{z})$. Therefore, $\mathcal{X}(\mathrm{D}(\mathbf{0})) \cap \mathrm{D}(\mathbf{z}) \neq \emptyset$.

Corollary 4.1. Let $\mathcal{C}$ be a binary code then $\mathcal{Z}_{\min }(\mathcal{C}) \subseteq \mathrm{L}(\mathcal{C})$, for any minimal test-set $\mathcal{Z}_{\text {min }}(\mathcal{C})$ obtained from $\mathcal{Z}(\mathcal{C})$.

Proof. Let $\mathcal{Z}_{\min }(\mathcal{C})$ be a minimal test-set of $\mathcal{C}$ obtained from $\mathcal{Z}(\mathcal{C})$, then every $\mathbf{z} \in \mathcal{Z}_{\text {min }}(\mathcal{C})$ satisfies that $\mathcal{X}(\mathrm{D}(\mathbf{0})) \cap \mathrm{D}(\mathbf{z}) \neq \emptyset$. Thus, by Theorem 4.5, we obtained the required result. 
Algorithm 2 gives the set of leader codewords $\mathrm{L}(\mathcal{C})$ of a binary code $\mathcal{C}$. Furthermore, any minimal test-set $\mathcal{Z}_{\text {min }}$ is a subset of $\mathrm{L}(\mathcal{C})$. Thus, after performing redundancy elimination to $\mathrm{L}(\mathcal{C})$, a minimal test-set $\mathcal{Z}_{\min }$ can also be obtained.

Example 4.1. We use the same code of Example 3.1. Algorithm 2 returns L $(\mathcal{C})$ and $\mathrm{L}^{1}(\mathcal{C})$, in this case we obtained that both sets coincide. We describe below the set of leader codewords with 14 elements of the given binary code $\mathcal{C}$.

$$
\mathrm{L}(\mathcal{C})=\mathrm{L}^{1}(\mathcal{C})=\left\{\begin{array}{cc}
\mathbf{e}_{3}+\mathbf{e}_{4}+\mathbf{e}_{7}+\mathbf{e}_{8}, & \mathbf{e}_{2}+\mathbf{e}_{4}+\mathbf{e}_{6}+\mathbf{e}_{8}, \\
\mathbf{e}_{2}+\mathbf{e}_{3}+\mathbf{e}_{6}+\mathbf{e}_{7}, & \mathbf{e}_{1}+\mathbf{e}_{4}+\mathbf{e}_{5}+\mathbf{e}_{8} \\
\mathbf{e}_{1}+\mathbf{e}_{3}+\mathbf{e}_{5}+\mathbf{e}_{7}, & \mathbf{e}_{1}+\mathbf{e}_{2}+\mathbf{e}_{5}+\mathbf{e}_{6}, \\
\mathbf{e}_{4}+\mathbf{e}_{6}+\mathbf{e}_{7}+\mathbf{e}_{9}+\mathbf{e}_{10}, \mathbf{e}_{3}+\mathbf{e}_{6}+\mathbf{e}_{8}+\mathbf{e}_{9}+\mathbf{e}_{10}, \\
\mathbf{e}_{2}+\mathbf{e}_{7}+\mathbf{e}_{8}+\mathbf{e}_{9}+\mathbf{e}_{10}, \mathbf{e}_{2}+\mathbf{e}_{3}+\mathbf{e}_{4}+\mathbf{e}_{9}+\mathbf{e}_{10}, \\
\mathbf{e}_{1}+\mathbf{e}_{5}+\mathbf{e}_{6}+\mathbf{e}_{7}+\mathbf{e}_{8}+\mathbf{e}_{9}+\mathbf{e}_{10} \\
\mathbf{e}_{1}+\mathbf{e}_{3}+\mathbf{e}_{4}+\mathbf{e}_{5}+\mathbf{e}_{6}+\mathbf{e}_{9}+\mathbf{e}_{10} \\
\mathbf{e}_{1}+\mathbf{e}_{2}+\mathbf{e}_{4}+\mathbf{e}_{5}+\mathbf{e}_{7}+\mathbf{e}_{9}+\mathbf{e}_{10} \\
\mathbf{e}_{1}+\mathbf{e}_{2}+\mathbf{e}_{3}+\mathbf{e}_{5}+\mathbf{e}_{8}+\mathbf{e}_{9}+\mathbf{e}_{10}
\end{array}\right\}
$$

Note that the only nonzero codeword of $\mathcal{C}$ that is missing in $\mathrm{L}(\mathcal{C})$ is the codeword $\mathbf{y}=(1,1,1,1,1,1,1,1,0,0)$ of weight 8 . This result is consistent with the fact that the covering radius of $\mathcal{C}$ is $\rho(\mathcal{C})=3$, as shown in Example 3.1, and the statement of Theorem 4.3 where we proved that the weight of a leader codeword is always less or equal to $2 \rho(\mathcal{C})+1=7$.

$$
\mathcal{C}=\left\{\begin{array}{c}
(0,0,0,0,0,0,0,0,0,0),(1,0,0,0,1,1,1,1,1,1),(0,1,0,0,0,0,1,1,1,1), \\
(1,1,0,0,1,1,0,0,0,0),(0,0,1,0,0,1,0,1,1,1)(1,0,1,0,1,0,1,0,0,0), \\
(0,1,1,0,0,1,1,0,0,0),(1,1,1,0,1,0,0,1,1,1),(0,0,0,1,0,1,1,0,1,1), \\
(1,0,0,1,1,0,0,1,0,0),(0,1,0,1,0,1,0,1,0,0),(1,1,0,1,1,0,1,0,1,1) \\
(0,0,1,1,0,0,1,1,0,0),(1,0,1,1,1,1,0,0,1,1),(0,1,1,1,0,0,0,0,1,1), \\
(\mathbf{1}, \mathbf{1}, \mathbf{1}, \mathbf{1}, \mathbf{1}, \mathbf{1}, \mathbf{1}, \mathbf{1}, \mathbf{0}, \mathbf{0})
\end{array}\right\}
$$

In the following table we present the computation results of a binary Golay code and a binary $\mathrm{BCH}$ code.

\begin{tabular}{|l|c|c|}
\hline & {$[23,12]$ Golay code } & {$[21,12]$ BCH code } \\
\hline Codewords $\left(2^{k}\right)$ & 4096 & 4096 \\
\hline Cosets $\left(2^{n-k}\right)$ & 2048 & 512 \\
\hline Leader codewords $(|\mathrm{L}(\mathcal{C})|)$ & 253 & 549 \\
\hline$\left|\mathrm{L}^{1}(\mathcal{C})\right|$ & 253 & 470 \\
\hline
\end{tabular}

Table 2. Number of codewords, number of cosets, number of leader codewords and the cardinality of $\left|\mathrm{L}^{1}(\mathcal{C})\right|$ of the $[23,12,7]$ binary Golay code and the $[21,12,5]$ binary BCH code.

Therefore, we show an example where the subsets $\mathrm{L}(\mathcal{C})$ and $\mathrm{L}^{1}(\mathcal{C})$ agree (this is not a surprise since the Golay code is a perfect code) and an example where the 
set $\mathrm{L}^{1}(\mathcal{C})$ is smaller than $\mathrm{L}(\mathcal{C})$. Also, note that both codes have the same number of codewords but the Golay code has four times the number of cosets of the $\mathrm{BCH}$ code. On the other hand, the number of leader codewords is less in the Golay code.

Lemma 4.1. If $\mathcal{C}$ is a perfect code, then $|\mathrm{L}(\mathcal{C})|=\left|\mathrm{L}^{1}(\mathcal{C})\right|$.

Proof. If $\mathcal{C}$ is a perfect code then every coset of $\mathcal{C}$ has a unique coset leader. That is, $\mathcal{N}=\mathrm{CL}(\mathcal{C})$. Recall that the only difference between the sets $\mathrm{L}^{1}(\mathcal{C})$ and $\mathrm{L}(\mathcal{C})$ is that the component $\mathbf{n}_{2}$ of any element $\mathbf{b}=\mathbf{n}_{1}+\mathbf{n}_{2}+\mathbf{e}_{i}$ from $\mathrm{L}^{1}(\mathcal{C})$ is required to belong to $\mathcal{N} \subseteq \mathrm{CL}(\mathcal{C})$. But in this case this difference doesn't exists.

\section{Implementations}

All the algorithms of this paper have been implemented and added to the collection of programs and procedures GBLA_LC (Gröbner Basis by Linear Algebra and Linear Codes). This framework consist of various files written in the GAP [7] language and included in GAP's package GUAVA 3.10. Also during the Google Summer of code of 2013 (http://www.google-melange.com/gsoc/homepage/google/gsoc2013) the student Verónica Suaste (CIMAT, México) implemented Algorithm 1 for inclusion in Sage 15. The code is published at http://trac.sagemath.org/ticket/14973 and it will be included in next releases of Sage.

\section{Aknowledgements}

The authors gratefully acknowledge the helpful comments and suggestions of the editor and the anonymous referees which contribute to a considerable improvement of this work.

\section{References}

[1] A. Barg. Complexity issues in coding theory. In Handbook of coding theory, Vol. I, II, pages 649-754. North-Holland, Amsterdam, 1998.

[2] E. R. Berlekamp, R. J. McEliece, and Henk C. A. van Tilborg. On the inherent intractability of certain coding problems. IEEE Trans. Information Theory, IT24(3):384-386, 1978.

[3] M. Borges-Quintana, M. A. Borges-Trenard, I. Márquez-Corbella, and E. MartínezMoro. An algebraic view to gradient descent decoding. In Information Theory Workshop (ITW), pages 1-4. IEEE, 2010.

[4] M. Borges-Quintana, M. A. Borges-Trenard, and E. Martínez-Moro. A Gröbner representation for linear codes. In Advances in coding theory and cryptography, volume 3 of Ser. Coding Theory Cryptol., pages 17-32. World Sci. Publ., Hackensack, NJ, 2007.

[5] M. Borges-Quintana, M. A. Borges-Trenard, and E. Martínez-Moro. On a Gröbner bases structure associated to linear codes. J. Discrete Math. Sci. Cryptogr., 10(2):151-191, 2007.

[6] J. Bruck and M. Naor. The hardness of decoding linear codes with preprocessing. IEEE Trans. Inform. Theory, 36(2):381-385, 1990.

[7] The GAP Group. GAP - Groups, Algorithms, and Programming, Version 4.12, 2009. 
November 4, 2014 4:46 WSPC/INSTRUCTION FILE CosetLeaders

[8] T. Helleseth, T. Klove, and V.I. Levenshtein. Error-correction capability of binary linear codes. Information Theory, IEEE Transactions on, 51(4):1408 - 1423, april 2005.

[9] W. Cary Huffman and Vera Pless. Fundamentals of error-correcting codes. Cambridge University Press, Cambridge, 2003.

[10] R.P. Kurshan and N.J.A. Sloane. Coset analysis of reed muller codes via translates of finite vector spaces. Information and Control, 20(5):410 - 414, 1972.

[11] F. J. MacWilliams and N. J. A. Sloane. The Theory of Error-Correcting Codes. Elsevier/North Holland, Amsterdam, 1977.

[12] I. Márquez-Corbella and E. Martínez-Moro. Algebraic structure of the minimal support codewords set of some linear codes. Adv. Math. Commun., 5(2):233-244, 2011.

[13] J. L. Massey. Minimal codewords and secret sharing. In Proceedings of the 6th Joint Swedish-Russian International Workshop on Information Theory, pages 246-249. 1993.

[14] Havard Raddum. On the computation of coset leaders with high Hamming weight. Discrete Mathematics, 274(1-3):213 - 231, 2004.

[15] William Stein. Sage: Open Source Mathematical Software. The Sage Group, 2008. http://www . sagemath.org.

[16] K. Yasunaga and T. Fujiwara. On correctable errors of binary linear codes. Information Theory, IEEE Transactions on, 56(6):2537 -2548, june 2010. 